Volume 10, No.2, March - April 2021

International Journal of Advanced Trends in Computer Science and Engineering

Available Online at http://www.warse.org/IJATCSE/static/pdf/file/ijatcse1081022021.pdf

https://doi.org/10.30534/ijatcse/2021/1081022021

\title{
A Comparative Analysis of Search Engine Ranking Algorithms
}

\author{
Murtaza Ahmed Hingoro ${ }^{1}$, Haque Nawaz $^{2, *}$ \\ ${ }^{1}$ BSCS Student, Sindh Madressatul Islam University, Karachi, Sindh, Pakistan, murtazaahmed842@gmail.com \\ ${ }^{2}$ Sindh Madressatul Islam University, Karachi, Sindh, Pakistan, hnlashari@smiu.edu.pk
}

\begin{abstract}
Ranking Algorithm is the most proper way of positioning on a scale. As the information and knowledge on the internet are increasing every day. The search engine's ability to deliver the most appropriate material to the customer. It is more and more challenging without even any assistance in filtering through all of it. However, searching what user requires is extremely difficult. In this research, an effort has been made to compare and analyze the most popular and effective search engines. The keywords were used in uniform resource locator like, title tag, header, or even the keyword's resembles to the actual text. The page rank algorithm computes a perfect judgment of how relevant a webpage is by analyzing the quality and calculating the number of links connected to it. In this study the keyword relevancy and time response were used for search engines and observed the results. It is observed that the google search engine is faster than the bing and youtube, and after all, bing is the best search engine after google. Moreover, youtube is the fastest search engine in terms of video content search. The google results were found more accurate. However, it is better than all of the search engines.
\end{abstract}

Key words: Ranking Algorithm, Google Search Engine, Bing Search Engine, YouTube Search Engine, Comparative Analysis.

\section{INTRODUCTION}

Today, the worldwide web is one of the biggest source of information in the digital world since its birth many websites has been registered according to the current figure of the google there are approximately 4.2 billion web pages currently active [1]. As a result, search engines have a great difficulty discovering appropriate information online.

Here, page rank algorithms play an important role to rank the most relevant content from the web pages. The page rank set of rules become without a doubt evolved at Stanford University with the aid of using Larry Page in 1996 as a part of studies for a brand new search engine [2]. A ranking algorithm is the most proper way of positioning on a scale. Many ranking algorithm are developed rather than one. Therefore, it provides the all users, even more up-to-date information.

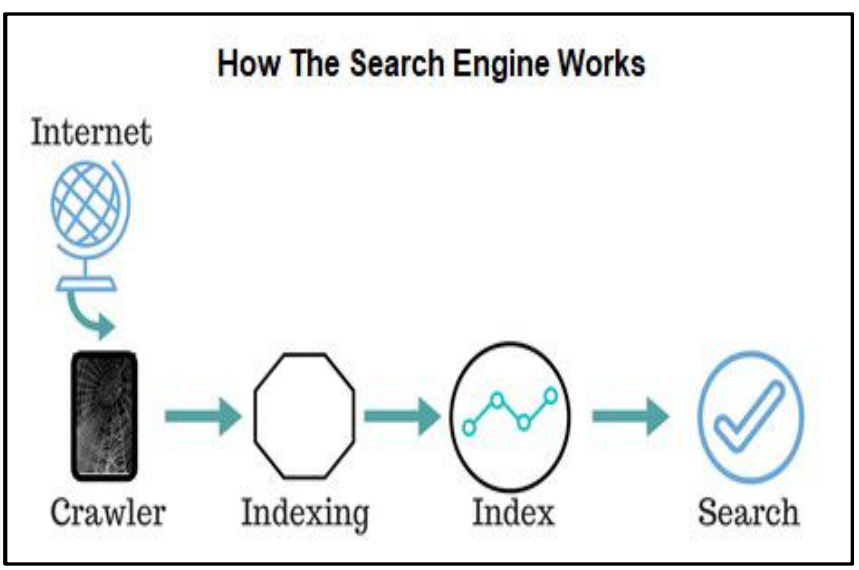

Figure 1: Shows working of Search Engine

The algorithm of search engines look at a lot of factors, which include the keywords of the user's query about search, Title Tag, relevancy, and usability of the links, the sources, and user location and settings.

As the amount of knowledge available on the worldwide web increases nearly every day, the search engine's ability to deliver most appropriate material to the one who use is becoming more and more challenging, without any assistance in filtering through all of it, knowing specifically what you require may be extremely difficult. The aim of ranking algorithms is always to help you reach what you're searching for, by filtering across hundreds of millions of web sites in the data base in a couple of seconds to search its most important, usable results [3].

In this research, we have compared the different search engines. The rest of paper is organized in sections, section 1 highlights the introduction; section 2 presents the literature review; section 3 highlight the methodology; section 4 show the comparative analysis of google search engine; section 5 presents the comparative analysis of bing search engine; section 6 highlight the comparative analysis of youtube search engine; section 7 shows the comparison of search engine results and discussion; section 8 authors have concluded. 


\section{LITERATURE REVIEW}

The world wide web is growing rapidly and the work of search engines is increasing day by day. There are more than thousands of search engines available on the internet but there are some most popular search engines like google, youtube, and bing. They are popular because of their searching techniques of providing the most relevant result to the user. To figure which search engine is best previously many research has been made. A survey on the web page rank algorithm has been conducting by Mercy Paul [4] which concluded that the site with a lot of connections has a lot of sources that can help with retrieving. Providing a different technique to increase retrieving accuracy, when both information as well as the connection are combined.

$$
P R(u)=\sum_{v \in B_{u}} \frac{P R(v)}{L(v)},
$$

Each algorithm has its own advantages and disadvantages. Similarly ranking algorithms also has some limitations in some particular terms. In another research work comparison and analyses of page ranking algorithms is done by Dilip Kumar Sharma [5] his research suggests that established approaches involve drawbacks, especially in terms of processing time, consistency of outcomes, relevance of result obtained, and usefulness of findings. As a result, an effective algorithm of page ranking should be able to face these hurdles while being compatible with worldwide website development requirements.

As the need, the user to retrieve the most related information page rank algorithm does web mining to retrieve information. Here is another research by Ashutosh Kumar Singh [6], The primary aim of this analysis is to make a comparison the different Page Rank-based techniques being used data extraction. For each execution of the search ranking algorithm, this indicates a timeframe [7].

\section{METHODOLOGY}

The ranking is an important position on a scale, there are different search engines that use different Algorithms to examine numerous factors to decide which web site is maximum applicable to a user's seek query. Search Engines that authors have considered in this research paper like google, bing, and youtube, etc. use different elements and aspects in their algorithm to determine relevance query generally which referred to as "Signals". The use of such a phrase with in uniform resource locator, title tag, heading or the similarity of the keywords to the beginning of the text are all examples of such signals. These included items like connections from these other pages, the importance of the site from which the connection originally comes, the link's content, as well as the other website on which the connection refers.
Google claims to use almost 200 signals in their rankings algorithms, although some claim to use just a few. Mostly in the general case, the below is a generalized representation of formula of ranking of the page:

In which the site ranking charge for an online site $\mathrm{u}$ is calculated by the page rank values for each online site $\mathrm{v}$ in the package $\mathrm{Bu}$ (this set of principles includes all sites connecting to the online site $u$ ), separated by the number of urls from site $\mathrm{v}$ [8]. To calculate the web page rank there is also a general formula available that is:

Where:

$$
\begin{gathered}
P R(A)=\text { PageRankofPageA } \\
T 1 \ldots T n=\text { Linktowardstheallpages. } \\
P R(T i)=\text { PageRankofthepageTi } \\
C(T i)=\text { TherangeofpagestowardswhichTihyperlink } \\
d=\text { DampingFactorwhichcanbe } 0 \text { and } 1
\end{gathered}
$$

\subsection{Pseudo Code}

Here is the pseudo code for the rankings algorithm implementation [9]. That is being used to illustrate the application of the general formula:

1. Working of Page Rank procedure where $\mathrm{G}$ is in-link file and iterations are number of the iterations.

2. Consider Damping factor $\mathrm{D}=0.85$.

3. From G get the out link count hash, symbol "oh".

4. From $\mathrm{G}$ get the in link hash, symbol "ih".

5. From $\mathrm{G}$ get the total number of the pages $\mathrm{N}$.

6. Graph have worked same for all $P$.

7. Initialize the page rank $1 / \mathrm{N}->$ opg[p].

8. End of for loop.

9. Apply while condition, iteration $>0$ then do.

10. The value of $d p$ is ' $O$ '.

11. Perform same operation for all $\mathrm{p}$ with no outlinktodo

12. Get the page rank opg $[\mathrm{p}] / \mathrm{N} * \mathrm{~d}+\mathrm{dp}->\mathrm{dp} \&$ calculate the pages without link.

13. End of for loop.

14. For all $\mathrm{p}$ Perform the same operation.

15. Have the page rank form random jumps $n p g[p]$

$=\mathrm{dp}+1-\mathrm{d} / \mathrm{N}$

16. Perform same operation for all $p$ in the ih $[\mathrm{p}]$

17. Generate page rank form the in links

18. End of for loop.

19. Enhance the page rank by npg $=$ opg.

20. Iteration is starting from $=$ iteration -1 .

21. End for loop.

22. Finish the complete procedure and generate result.

\subsection{Principle of Page Rank}

The page rank algorithm calculates a pure judgement about how significant a website is by examining the reliability and calculating the lot of connections leading to it [10]. The fundamental premise is that the more important websites are more likely to receive additional connections from the other sited. 


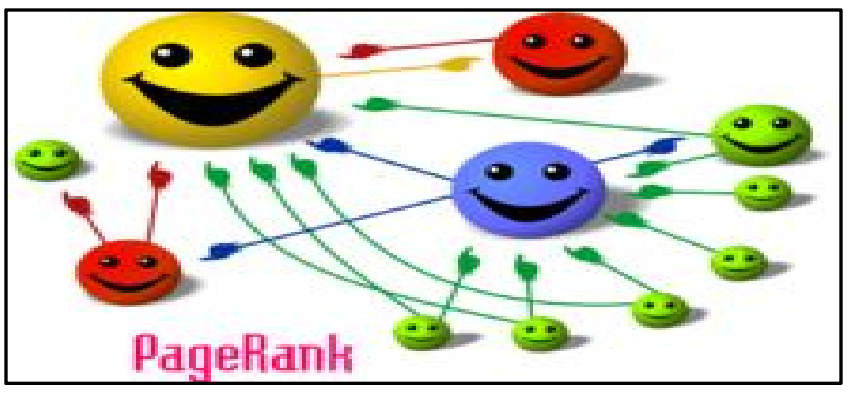

Figure 2: Shows the Concept of Page Rank

\subsection{Flow Chart of Page Rank}

Here is the flowchart which generally represents how a page rank algorithm crawl on the internet to rank the pages on the basis of relevancy.

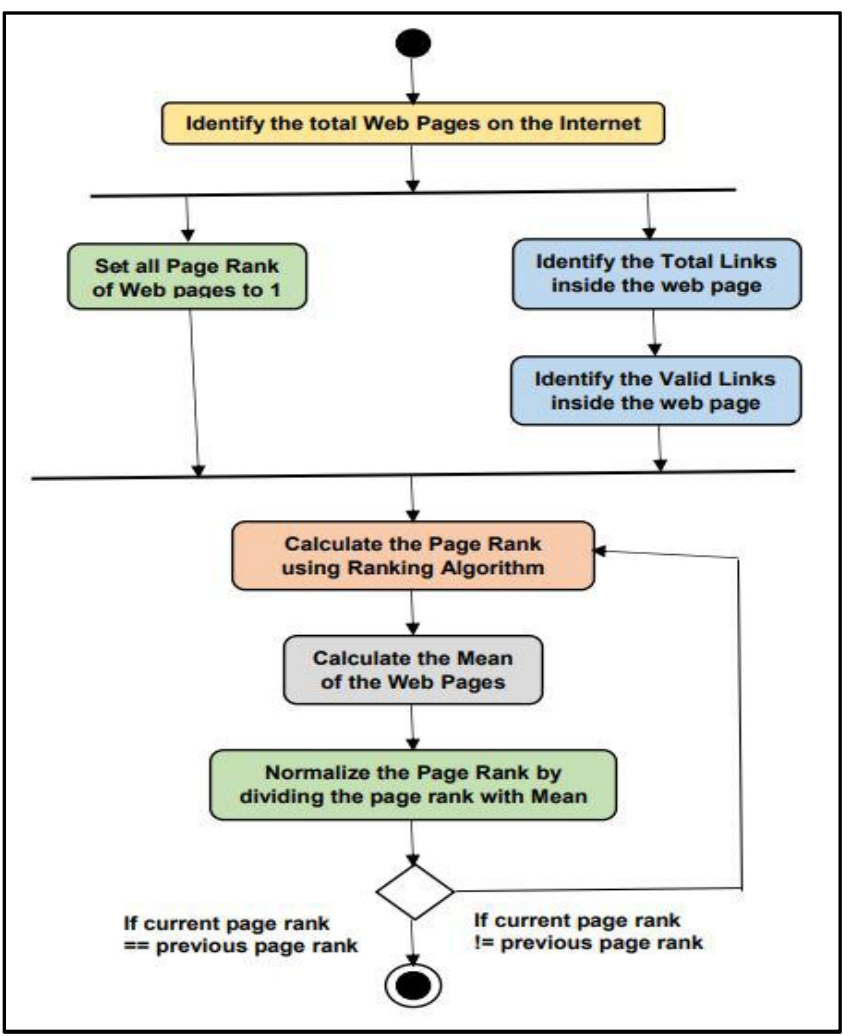

Figure 3: Shows the Working Flow of Page Rank

\section{COMPARATIVE ANALYSIS OF GOOGLE SEARCH ENGINE}

With billions of web pages on the internet, without any assistance in filtering through all of it, knowing specifically what you require may be extremely difficult [11]. Google rating machine is designed to do simply that in a couple of seconds, their Search engine sorts through millions of web sites to discover the most important, useful effects, and presents those in a way that helps everyone to reach what they are searching for [12]. This ranking algorithms are constructed up of a sequence of algorithms rather than just one. To give the individual even more up-to-date details possible. The keywords in the participant's question are one of the many variables recognized by search engine rankings about search, title tag, relevancy, and usability of the links, the sources, and your location and settings [13]. So let's have a look on google search engine.

\section{Google}

Q Search Google or type a URL

Figure 4: Shows the Google Search Engine

\subsection{Comparison of Google Search Engine}

Table 1: Observation of Google Search Engine

\begin{tabular}{|l|l|l|r|}
\hline S. No. & Keywords & Time Taken & Total Results \\
\hline 1 & Information & 0.71 & $16910,000,000$ \\
\hline 2 & Nature & 1.10 & $9890,000,000$ \\
\hline 3 & Algorithm & 0.64 & $421,000,000$ \\
\hline 4 & Physics & 0.60 & $483,000,000$ \\
\hline 5 & Dragon & 0.70 & $1260,000,000$ \\
\hline
\end{tabular}

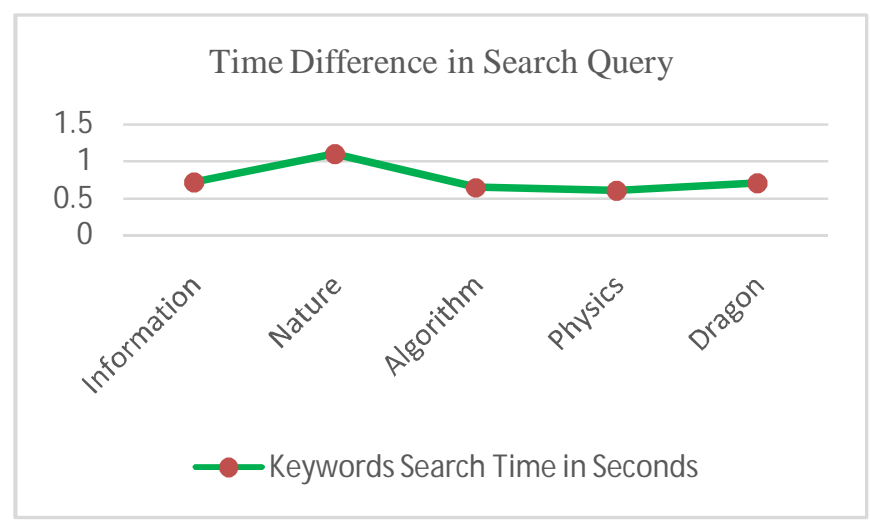

Figure 5: Show Time Difference in Google Algorithm

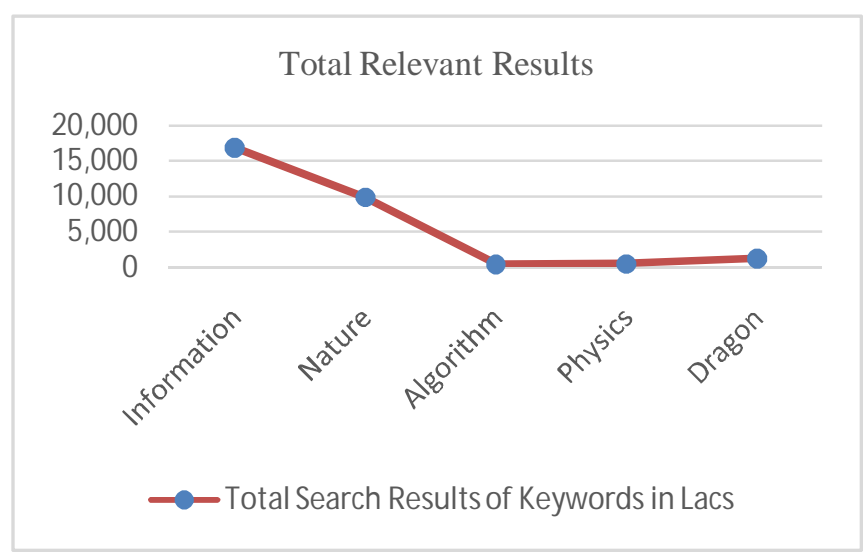

Figure 6: Show Relevant Results by Google Algorithm 


\section{COMPARATIVE ANALYSIS OF BING SEARCH ENGINE}

Bing is also one of the most popular search engines owns and operated by microsoft. Bing typically offers quite a few seek services, such as web, video, image, and map seek products. It is advanced the usage of ASP.NET [14]. Bing has an instant answer feature that provides quick information to the user about sports, finance, health information, flight tracking, package tracking and tracing, Advanced computations, and many more [15]. Bing rewards became a loyalty application released through microsoft in september 2010. It turned into just like in advance services, search perks, and bingcashback [16], which have been ultimately discontinue. So let's have a look at bing search engine.

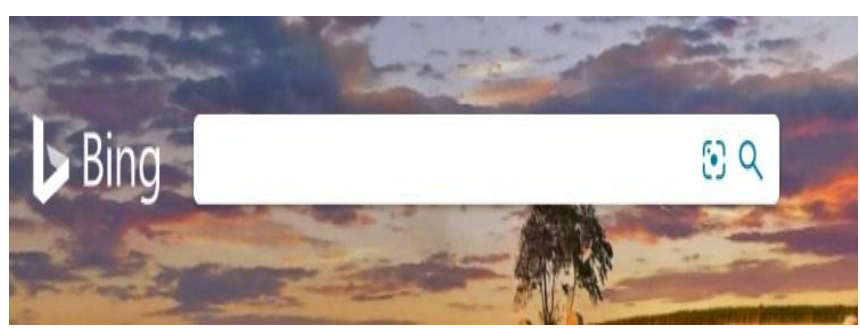

Figure 7: Shows the Bing Search Engine

Table 2:Observation of Bing Search Engine

\begin{tabular}{|l|l|l|r|}
\hline S. No. & Keywords & Time Taken & Total Results \\
\hline 1 & Information & 1.30 & $247,000,000$ \\
\hline 2 & Nature & 0.85 & $120,000,000$ \\
\hline 3 & Algorithm & 0.90 & $50,000,000$ \\
\hline 4 & Physics & 1.30 & 88400,000 \\
\hline 5 & Dragon & 1.35 & $103,000,000$ \\
\hline
\end{tabular}

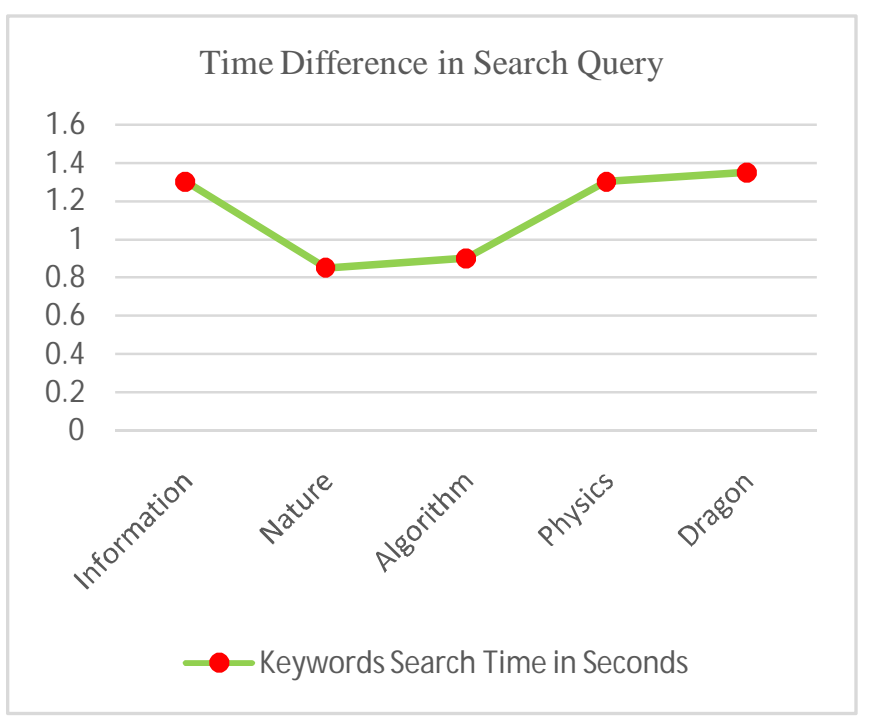

Figure 8: Show Time Difference in Bing Algorithm

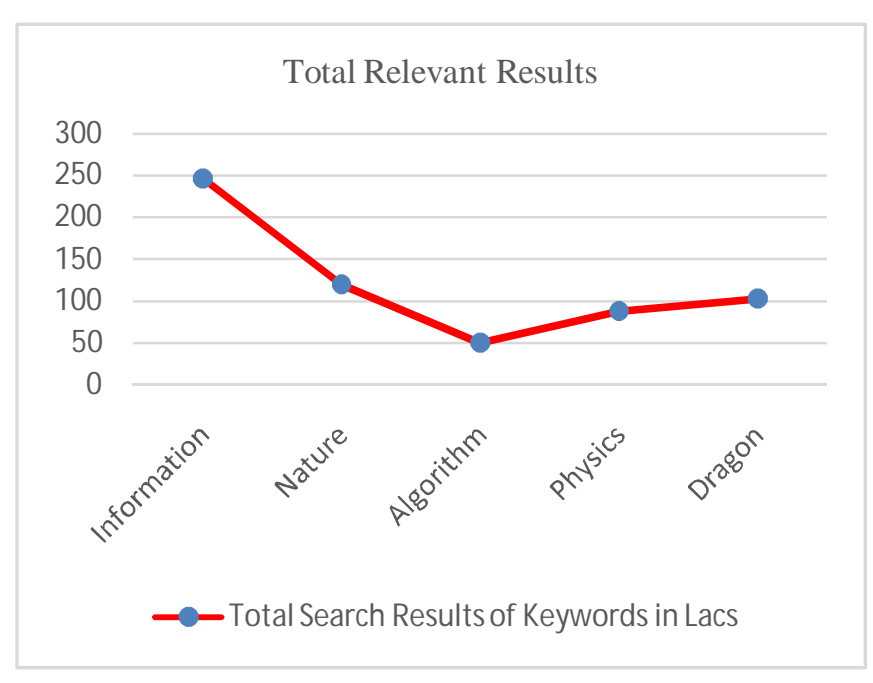

Figure 9: Show Relevant Results by Bing Algorithm

\section{COMPARATIVE ANALYSIS OF YOUTUBE SEARCH ENGINE}

The second biggest and maximum used seek engine within side the global is youtube. A video search engine is a web-based search engine that scans the world wide web for streaming video [16]. Most video search engines analyze external cloud videos, while others allow users to publish and host videos according to their personal servers [18]. Mostly search bars like google and yahoo additionally permit customers to look through video layout kind and through the duration of the clip. The results of video seek are normally observed from a preview overview including its stream [19].Video search engines, such as google, are definitely desktop software intended to find films stored on modern systems, whether on web server or in storage systems attached to the similar computer. Multimedia indexing may be used to perform similar scans [20], that's also capable of extracting content from multimedia material and storing it as data about the data, which can be monitored by google. The use of those engines like google is the growing introduction of the audiovisual content material and they want to manipulate that correctly. Owing to the vast numbers of archives as well as the life of a conceptual void, the digital technology of multimedia material and the established order of the network has resulted in substantial portions of video recordings being saved in enormous libraries, whose recovery can be difficult [21]. So let's have a look on youtube search engine.

\section{You Tube"}

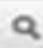

Figure 10: Shows the YouTube Search Engine 
Table 3:Observation of YouTube Search Engine

\begin{tabular}{|l|l|l|r|}
\hline S. No. & Keywords & Time Taken & Total Results \\
\hline 1 & Information & 0.20 & $5110,000,000$ \\
\hline 2 & Nature & 0.50 & $1340,000,000$ \\
\hline 3 & Algorithm & 0.35 & 3430,000 \\
\hline 4 & Physics & 0.31 & $36,000,000$ \\
\hline 5 & Dragon & 0.45 & $186,000,000$ \\
\hline
\end{tabular}

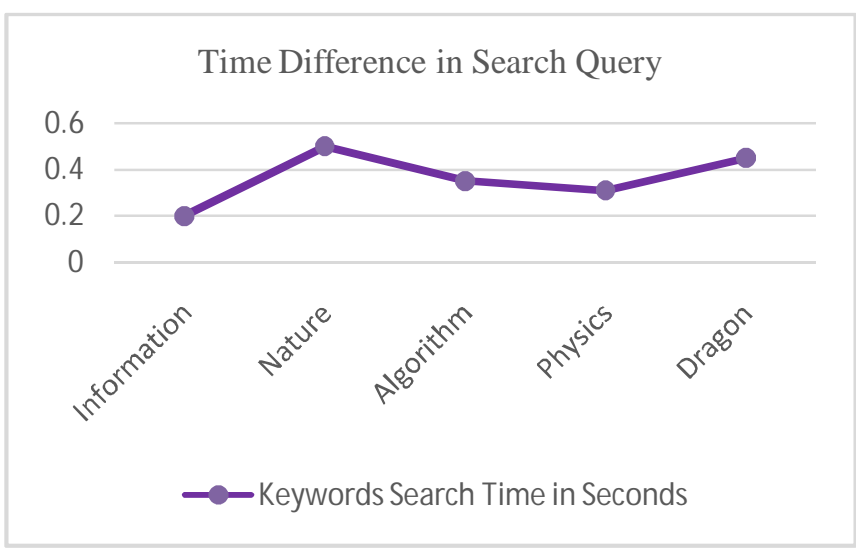

Figure 11:Show Time Difference in YouTube Algorithm

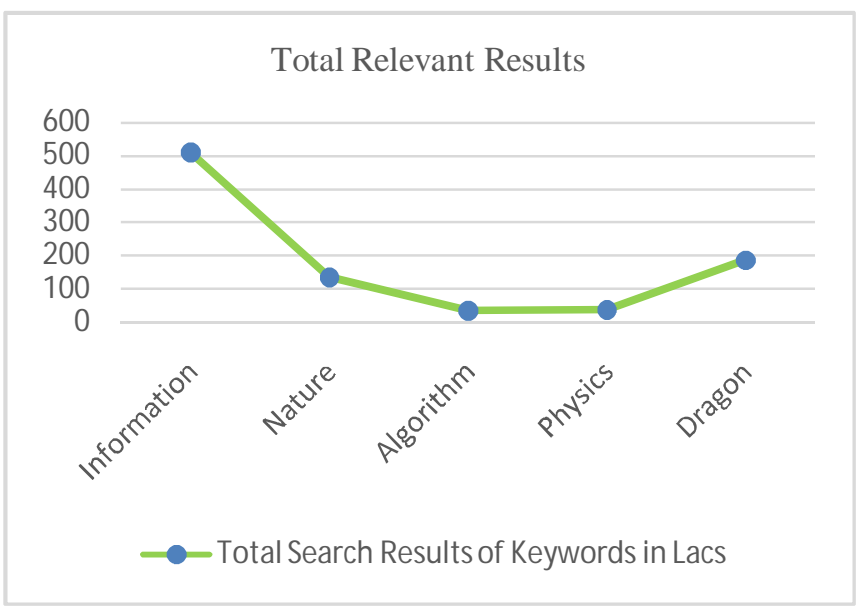

Figure 12: Show Relevant Results by YouTube Algorithm

\section{COMPARISON OF SEARCH ENGINE RESULTS AND DISCUSSION}

After doing a comparative analysis on the world three best and most popular search engines on the basis of their page rank algorithm, this research concluded that YouTube has the most fast ranking algorithm in the world but it considers only one factor of information that is video streaming. If you consider the different factor of information author conclude that The page ranking algorithm of the google is the fastest than the rest of the search engines because its search engine algorithms look at a lot of factors, which include the keywords of user's query about search, title tag, relevancy, and usability of the links, the sources, and your location and settings and in last user got bing that is still best search engine after the google. Its fast results and accurate result made it better than the rest of the search engines in the world. Unfortunately, bing does not have the best page ranking factor so it could defeat google's page rank algorithm

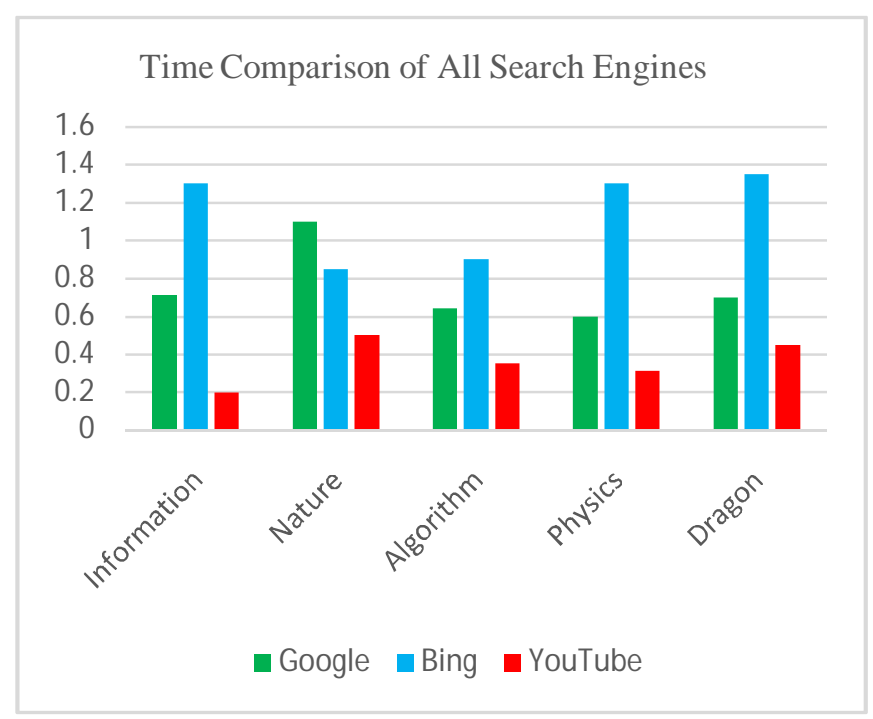

Figure 13: Shows Time Comparison of All Search Engines

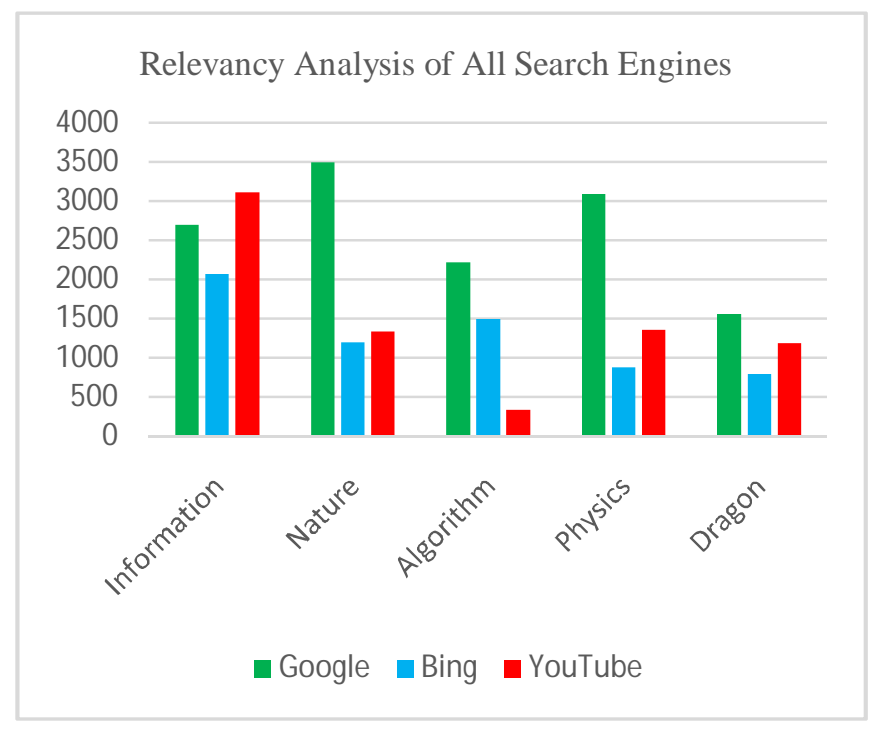

Figure 14: Shows Relevancy Analysis of all Search Engines

\section{CONCLUSION}

The website page rank is set of rules of various SERPs which is very effective in providing relevant information to the user and it is the world's fastest working algorithm. The algorithm used by google enhances the quality of search and this ranking algorithm of google can be used by other search engines to improve their efficiency of page ranking as per user's need. Every set of rules has its merits and demerits. In this research, 
we have analyzed the keyword relevancy and time response for all search engines and observed the results. The google search engine is faster than the bing and youtube search engines and after all, there is bing that is the best search engine after google and youtube is the fastest search engine in terms of video content search. Moreover, the google results were found more accurate. However, it is better than all of the search engines.

\section{ACKNOWLEDGEMENT}

Authors would like to thank the anonymous reviewers for their valuable comments and suggestions which certainly helped in the improvement of the manuscript. Lastly, they are grateful to the Editor of "IJATCSE" for providing a platform for publishing the manuscript.

\section{REFERENCES}

[1] D. Sharma, R. Shukla, A. K. Giri, and S. Kumar, "A Brief Review on Search Engine Optimization," in 9th International Conference on Cloud Computing, Data Science Engineering (Confluence), IEEE, Noida, India, 2019, pp. 687-692.

[2] D. K. Sharma and A. K. Sharma, "A Comparative Analysis of Web Page Ranking Algorithms," International Journal on Computer Science and Engineering, vol. 02, no. 08, pp. 2670-2676, 2010.

[3] C. Palihawadana and G. Poravi, "A Comparative Study of Link Analysis Algorithms," in 8th International Conference on Intelligent Systems, Modelling and Simulation (ISMS), Kuala Lumpur, Malaysia, 2018, pp. 100-104.

[4] M. PaulSelvan, A. Chandra Sekar, and A.PriyaDharshini "Survey on Web Page Ranking Algorithms" International Journal of Computer Applications, vol. 41, no. 19, pp. 1-7, 2012.

[5] R. Sardhara and K. I. lakhataria, "A flowchart to reduce mutual reinforcement effect on web page ranking based on web strucuture mining," in 3rd International conference on Electronics, Communication and Aerospace Technology (ICECA), IEEE, Coimbatore, India, Jun. 2019, pp. 34-38.

[6] A. K. Singh, and R. Kumar, "A Comparative Study of Page Ranking Algorithms for Information Retrieval" International Journal of Computer and Information Engineering, pp. 926-937, 2009.

[7] S. Irfan and S. Ghosh, "A Review on Different Ranking Algorithms," in International Conference on Advances in Computing, Communication Control and Networking (ICACCCN), Greater Noida, India, 2018, pp. 86-90.

[8] F. Zhan et al., "An efficient alternative to personalized page rank for friend recommendations," in 15th IEEE Annual Consumer Communications \& Networking Conference, IEEE, Las Vegas, NV, USA, 2018, pp. 1-2.

[9] K. Dai, "PageRank Lecture Note" June, 22, 2009.

[10] R. Sardhara and K. I. Lakhataria, "An Improved PageRank Algorithm Based on Reachability to Reduce Mutual Reinforcement Effect," in 10th
International Conference on Computing, Communication and Networking Technologies (ICCCNT), Kanpur, India, Jul. 2019, pp. 1-6, doi: 10.1109/ICCCNT45670.2019.8944481.

[11] S. Irfan and S. Ghosh, "Analysis Challenges of Web Ranking Algorithms," in 4th International Conference on Computing Communication and Automation (ICCCA), IEEE, Greater Noida, India, 2018, pp. 1-3.

[12] Y. Tenget al., "Application Of Ant Colony Algorithm In Crawler," in IEEE 3rd Advanced Information Technology, Electronic and Automation Control Conference (IAEAC), IEEE, Chongqing, China, 2018, pp. 2195-2198.

[13] M. Usha and N. Nagadeepa, "Combined two phase page ranking algorithm for sequencing the web pages," in 2nd International Conference on Inventive Systems and Control (ICISC), IEEE, Coimbatore, India, 2018, pp. 876-880.

[14] S. Suri, A. Gupta, and K. Sharma, “Comparative Study of Ranking Algorithms," in International Conference on Computing, Electronics \& Communications Engineering (iCCECE), London, United Kingdom, 2019, pp. 73-77.

[15] D. Vandic, S. Aanen, F. Frasincar, and U. Kaymak, "Dynamic Facet Ordering for Faceted Product Search Engines," IEEE Transactions On Knowledge And Data Engineering, vol. 29, no. 5, pp. 1004-1016, 2017.

[16] R. Sardhara and K. I. lakhataria, "Impact of Different Domain Inlink, Outlink and Rechability on Relevance of Web Page Using Correlation," in International Conference on Intelligent Computing and Control Systems (ICCS), IEEE, Madurai, India, 2019, pp. 755-759.

[17] M. K. Mittal, N. Kirar, and J. Meena, "Implementation of Search Engine Optimization : Through White Hat Techniques," in International Conference on Advances in Computing, Communication Control and Networking (ICACCCN), IEEE, Greater Noida (UP), India, 2018, pp. 674-678.

[18] A. Tiwari and S. Chaturvedi, "Optimized Technique for Ranking Webpage on Search Engine Optimization," in 2nd International Conference on Micro-Electronics and Telecommunication Engineering (ICMETE), IEEE, Ghaziabad, India, 2018, pp. 107-110.

[19] U. Rathod, A. Pavate, and V. Patil, "Product Rank Based Search Engine for E-Commerce," in 3rd International Conference for Convergence in Technology (I2CT), IEEE, Pune, India, Apr. 2018, pp. $1-5$.

[20] M. A. Hasson, S. F. Lu, and B. A. Hassoon, "Scientific Research Paper Ranking Algorithm PTRA: A Tradeoff between Time and Citation Network," Applied Mechanics and Materials, vol. 551, pp. 603-611, 2014.

[21] B. Joglekar, R. Bhatia, S. Jayaprakash, K. Raina, and S. Mulchandani, "Search Engine Optimization Using Unsupervised Learning," in 5th International Conference On Computing, Communication, Control And Automation (ICCUBEA), Pune, India, 2019, pp.1-5. 